\title{
Hysterectomy for Recurrent/Residual Cervical Cancer Following Definitive Radiotherapy
}

\author{
TADAHARU NAKASONE, YUSUKE TAIRA, YUKO SHIMOJI, YOSHIHISA ARAKAKI, \\ TOMOKO NAKAMOTO, TAKUMA OOYAMA, WATARU KUDAKA, ITOMI KANESHIMA, \\ KUMIKO NISHIHIRA, KEIKO MEKARU and YOICHI AOKI \\ Department of Obstetrics and Gynecology, Graduate School of Medicine, \\ University of the Ryukyus, Okinawa, Japan
}

\begin{abstract}
Background/Aim: Radical hysterectomy has been used for local recurrent or persistent (LR) cervical cancer after radiotherapy $(R T)$, but the rate of serious complications is high and tolerance is low. This study determined the efficacy, safety, and prognostic factors of adjuvant simple hysterectomy in LR cervical cancer post-RT. Patients and Methods: A total of 21 patients who underwent hysterectomy for LR cervical cancer post-RT in our Department between May 2007 and September 2018 were included in the study. Primary, definitive RT was performed. Histological response by definitive $R T$ in the extirpated uterus was classified on the basis of histological response criteria: effect (Ef) 0-3. Results: The 5-year overall survival (OS) and disease-free survival (DFS) rates were $51.9 \%$ and $50.1 \%$, respectively. Ef 1 was significantly associated with poorer prognosis compared to Ef 2 or Ef 3. Conclusion: Adjuvant hysterectomy could be a treatment of choice for LR cervical cancer post-RT.
\end{abstract}

Radiotherapy (RT) is an effective treatment for cervical cancer, and concurrent chemoradiotherapy (CCRT) is the standard treatment for locally advanced cervical cancer. However, residual (1-4) or subsequent local recurrence is 10$30 \%$, and these cases have poor prognosis. In Japan, best supportive care (BSC), chemotherapy for symptom relief, and pelvic exenteration or hysterectomy for central recurrence are the options for recurrence inside the radiation field of recurrence (5). Resection of local lesions alone might

This article is freely accessible online.

Correspondence to: Tadaharu Nakasone, Department of Obstetrics and Gynecology, Graduate School of Medicine, University of the Ryukyus, 207 Uehara Nishihara, Okinawa 903-0215, Japan. Tel: +81 988951177, Fax: +81 988951426, e-mail: miamiamia1229@ gmail.com

Key Words: Cervical cancer, radiotherapy, hysterectomy. improve prognosis, but results are controversial. Radical hysterectomy has been used for local recurrent or residual (LR) cervical cancer post-RT, but the rate of serious complications is high and tolerance is low $(6,7)$. Several studies have reported the usefulness of salvage simple total hysterectomy, and efforts have been made to reduce complications $(8,9)$. These studies have shown a 5-year overall survival (OS) of $49 \%-72 \%$ (6-8) and a 5-year disease-free survival (DFS) of $90 \%$ (9), with the rate of complications being $42 \%-44 \%$ in radial hysterectomy $(6,7)$, and $0 \%-14 \%$ in simple hysterectomy $(8,9)$.

This study determined the efficacy, safety, and prognostic factors of adjuvant simple hysterectomy in LR cervical cancer post-RT.

\section{Patients and Methods}

We included 21 patients who underwent hysterectomy for LR cervical cancer post-RT in our department between May 2007 and September 2018. We retrospectively reviewed their medical records for their background, clinicopathological factors, complications, and prognosis. Primary, definitive RT was performed as described previously (10). Briefly, a 50 Gy dose of whole-pelvic external beam RT was delivered in 25 fractions. A center shield $4 \mathrm{~cm}$ wide at the midline was used after delivery of a 40 Gy dose of RT. High-doserate intracavitary brachytherapy (HDR-ICBT) was delivered once per week at a fractional dose of 6 Gy one to three times at point A (total dose of 6-18 Gy). The cumulative linear quadratic equivalent dose (EQD2) was 62-65 Gy prescribed at point A (10). The CCRT regimen included $40 \mathrm{mg} / \mathrm{m}^{2}$ of cisplatin per week for patients with squamous cell carcinoma (SCC) (10) and $50 \mathrm{mg} / \mathrm{m}^{2}$ of cisplatin every 3 weeks and $50 \mathrm{mg} / \mathrm{m}^{2}$ paclitaxel per week for patients with non-SCC (11).

Follow-up examinations were performed every month until 3 months post-RT or post-CCRT. LR cervical cancer was diagnosed on the basis of biopsies with pathological assessment and computed tomography (CT) scans, positron emission tomography (PET), or magnetic resonance imaging (MRI) 3 months post-RT. Patients with residual viable cancer in the cervix without distant metastasis or obvious lymph node metastasis underwent total abdominal hysterectomy, bilateral salpingo-oophorectomy, and pelvic lymph node sampling. Ureteral stents were placed in all patients preoperatively. 
Table I. Histological response classification.

\begin{tabular}{|c|c|}
\hline Effect & Histological findings \\
\hline 0 & $\begin{array}{l}\text { No morphological change such as degeneration or necrosis is } \\
\text { observed in cancer cells and cancer tissue }\end{array}$ \\
\hline 1 & $1 / 3$ or more of cancer cells survive \\
\hline 2 & Less than $1 / 3$ cancer cells survive \\
\hline 3 & $\begin{array}{l}\text { Cancer cells are not recognized or unable to survive even if } \\
\text { they remain }\end{array}$ \\
\hline
\end{tabular}

Table II. Patient characteristics.

\begin{tabular}{lc}
\hline Variables & \\
\hline Median age (range) & $53(31-84)$ years \\
Histological subtype & \\
$\quad$ Squamous cell carcinoma & 14 \\
Adenocarcinoma & 7 \\
FIGO stage & 11 \\
IB2 & 8 \\
IIB & 2 \\
IIIB & 17 \\
Persistence or recurrence & 4 \\
Persistence & $10(0-51) \mathrm{mm}$ \\
Recurrence & 10 \\
Median tumor diameter at hysterectomy (range) & 6 \\
Primary treatment & 2 \\
P-CCRT & 3 \\
TP-CCRT & \\
TP-NAC, TP-EFCCRT & \\
Others & \\
\hline
\end{tabular}

FIGO: The International Federation of Gynecology and Obstetrics; PCCRT: concurrent chemoradiotherapy with cisplatin; TP-CCRT: concurrent chemoradiotherapy with paclitaxel and cisplatin; TP-NAC: neoadjuvant chemotherapy with paclitaxel and cisplatin; TP-EFCCRT: extended field concurrent chemoradiotherapy with paclitaxel and cisplatin.

Histological response by definitive RT in the extirpated uterus was classified on the basis of histological response criteria, as follows: effect (Ef) 0: no morphological changes, such as degeneration and necrosis, observed in cancer cells and cancer tissues; Ef 1: cancer cells that can survive in $\sim \geq 1 / 3$ of cancers; Ef 2: cancer cells that can survive in $<1 / 3$ of cancers; Ef 3 : cancer cells not recognized or thought to be unable to survive, even if present (Table I) (12).

All statistical analyses were performed using JMP software version 15.0 (SAS Institute, Cary, NC, USA). The Kaplan-Meier method and the log-rank test were used to assess the survival rate. $p<0.05$ was considered statistically significant.

All the patients provided their written informed consent regarding the treatment. All procedures performed in this study involving human participants were in accordance with the ethical standards of the institutional and/or national research committee and with the 1964 Declaration of Helsinki and its later amendments or

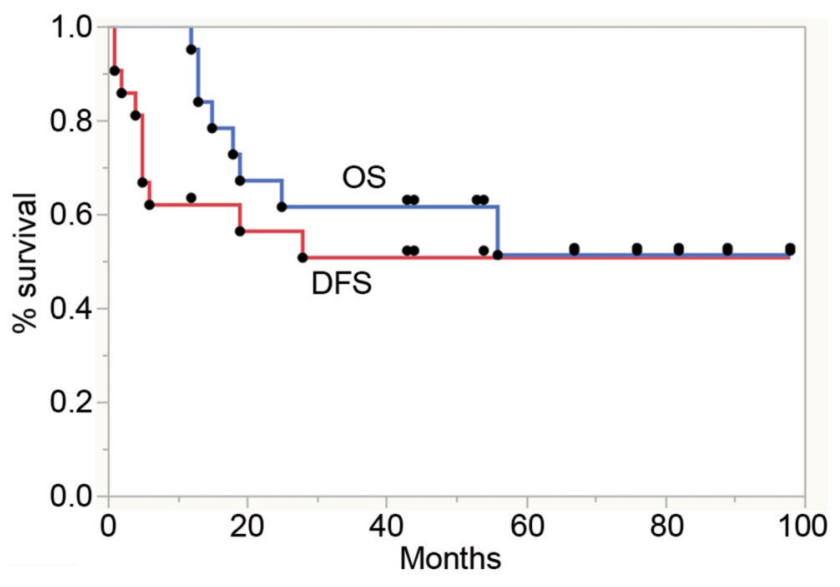

Figure 1. Kaplan-Meier curves for OS and DFS in the study population $(N=21)$. The 5-year $O S$ and DFS rates were $51.9 \%$ and $50.1 \%$, respectively. OS: Overall survival; DFS: disease-free survival.

comparable ethical standards. This retrospective study was approved by the Institutional Review Board of our university (\#1375 in Dec 10, 2018).

\section{Results}

Table II shows the patients' characteristics. The patients' median age was 55 years (range $=31-84$ years). The International Federation of Gynecology and Obstetrics (FIGO) stage distribution was as follows: 11 patients in stage IB2, 8 in stage IIB, and 2 in stage IIIB. In addition, 14 patients had SCC and 7 had adenocarcinoma. Persistent lesion was observed in 17 patients and recurrence in 4 patients. The median tumor diameter at hysterectomy was $10 \mathrm{~mm}$ (range=0$51 \mathrm{~mm}$ ). Serum SCC antigen was re-elevated in 5 patients $(23.8 \%)$ at hysterectomy. The median total surgery time was $182 \mathrm{~min}$ (range=121-333 $\mathrm{min}$ ), and the median total blood loss was $308 \mathrm{~mL}$ (range=62-685 ml). Perioperative complications included intraoperative bladder injury and postoperative pelvic abscess in 1 patient each $(9.5 \%)$. Postoperative adjuvant chemotherapy was administered to 3 patients (14.2\%) who had positive lymph nodes and close margins. Lymph node resection was performed in 5 patients with enlarged pelvic lymph nodes, revealing positive metastasis; 4 of these patients died of the disease.

The median OS and DFS were 43 months (range=9-98 months) and 19 months (range $=1-98$ months), respectively. The 5 -year OS and DFS rates were $51.9 \%$ and $50.1 \%$, respectively (Figure 1). With regard to prognostic factors, Ef 1 was significantly associated with poorer prognosis compared to Ef 2 or Ef 3. The 5-year OS was $88.9 \%$ in patients with Ef 2 or Ef 3 and $22.5 \%$ in patients with Ef $1(p=0.0129)$, while the 5year DFS was $78.8 \%$ in patients with Ef 2 or Ef 3 and $24.2 \%$ in patients with $\operatorname{Ef} 1$ ( $p=0.0172$ ) (Figure 2). Lymphovascular 

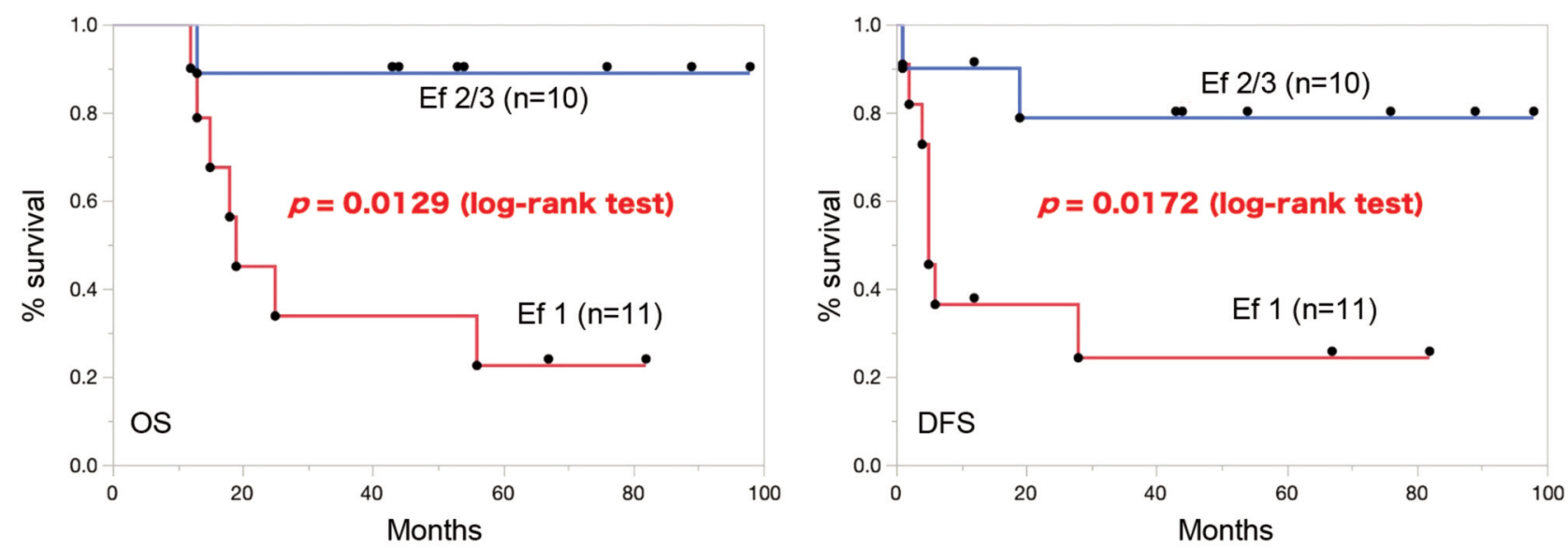

Figure 2. Kaplan-Meier curves for OS and DFS according to histological response (Ef 1 vs. Ef 2 or Ef 3). The 5-year OS was $88.9 \%$ in Ef 2 or Ef 3 patients and $22.5 \%$ in Ef 1 patients. The 5-year DFS was 78.8\% in Ef 2 or Ef 3 patients and $24.2 \%$ in Ef 1 patients.

Table III. Univariate analyses for overall survival and disease-free survival.

\begin{tabular}{|c|c|c|c|c|c|}
\hline Variables & No. & 5 -y overall survival $(\%)$ & $p$-Value & 5-y disease-free survival (\%) & $p$-Value \\
\hline Age (years) & & & 0.0876 & & 0.483 \\
\hline$\geq 56$ & 10 & 77.8 & & 60.0 & \\
\hline$<56$ & 11 & 22.2 & & 40.9 & \\
\hline Histological subtype & & & 0.591 & & 0.693 \\
\hline Squamous cell carcinoma & 14 & 63.6 & & 55.1 & \\
\hline Adenocarcinoma & 7 & 38.1 & & 42.9 & \\
\hline FIGO stage & & & 0.918 & & 0.931 \\
\hline IB2 & 11 & 62.5 & & 51.0 & \\
\hline IIB & 8 & 50.0 & & 50.0 & \\
\hline IIIB & 2 & 0 & & 50.0 & \\
\hline Recurrence or persistence & & & 0.542 & & 0.778 \\
\hline Recurrence & 4 & 66.7 & & 50.0 & \\
\hline Persistence & 17 & 52.1 & & 51.8 & \\
\hline Tumor diameter at surgery & & & 0.884 & & 0.928 \\
\hline$\geq 20 \mathrm{~mm}$ & 8 & 46.9 & & 50.0 & \\
\hline$<20 \mathrm{~mm}$ & 13 & 61.1 & & 51.3 & \\
\hline LVSI & & & 0.0368 & & 0.0570 \\
\hline Yes & 10 & 26.7 & & 30.0 & \\
\hline No & 11 & 90.0 & & 70.1 & \\
\hline Parametrial extension & & & 0.565 & & 0.721 \\
\hline Yes & 3 & 66.7 & & 66.7 & \\
\hline No & 18 & 45.4 & & 47.5 & \\
\hline LN metastasis & & & 0.195 & & 0.0836 \\
\hline Yes & 5 & 20.0 & & 20.0 & \\
\hline No & 16 & 70.0 & & 61.1 & \\
\hline Surgical margin & & & 0.414 & & 0.283 \\
\hline Positive & 1 & 0 & & 0 & \\
\hline Negative & 20 & 54.3 & & 53.2 & \\
\hline Histological response & & & 0.0129 & & 0.0172 \\
\hline Ef 1 & 11 & 22.5 & & 24.2 & \\
\hline Ef $2 / 3$ & 10 & 88.9 & & 78.8 & \\
\hline Serum SCC & & & 0.0717 & & 0.113 \\
\hline Elevated & 5 & 0 & & 0 & \\
\hline Normal & 16 & 66.7 & & 61.9 & \\
\hline
\end{tabular}

FIGO: The International Federation of Gynecology and Obstetrics; RT: radiotherapy; LVSI: lymphovascular involvement; LN: lymph node. 
space invasion (LVSI) was significantly associated with poor OS $(p=0.0368)$. In addition, age, FIGO stage, histological subtype, recurrence or persistence at hysterectomy, tumor size at hysterectomy, parametrial extension, lymph node metastasis, positive surgical margin, and elevated serum SCC were not significant prognostic factors (Table III). Although multivariate analysis showed no independent prognostic factor, univariate analysis showed that only histological response was significant in both OS and DFS.

Posthysterectomy, 10 patients had recurrence between 1 and 28 months, of which 8 had recurrence within 6 months. Locoregional recurrence occurred in 5 patients (pelvic lymph nodes, ureters, vaginal stumps), local and distant recurrence in 1 patient, and distant recurrence in 4 patients (Table IV). One patient showed lung metastasis 19 months posthysterectomy and underwent video-assisted thoracic surgery (VATS), with no evidence of disease (NED).

\section{Discussion}

Hysterectomy for LR cervical cancer post-RT or post-CCRT seems effective and tolerable. By 3 months post-RT, local residuals should be fully evaluated and hysterectomy considered. Previous studies have reported a 5-year OS of $49 \%-72 \%$ (6-8), which is consistent with our study. In addition, complications have been reported to develop in $42 \%-44 \%$ of patients who underwent radial hysterectomy (6, 7) but only in $0 \%-14 \%$ of patients who underwent simple hysterectomy $(8,9)$. Urinary tract injuries, fistulas, and bladder dysfunction are reported most as complications due to radial hysterectomy. Because the prognosis of patients treated with radial hysterectomy and simple hysterectomy is comparable, simple hysterectomy seems better because of reduced complications. Therefore, adjuvant hysterectomy post-RT is considered a treatment of choice.

Histological response could be a good indicator for prognosis. There are no reports on histological response criteria in gynecological cancers. In pancreatic cancer, the prognostic importance of histological response has been demonstrated (12). A pathological complete response and $<10 \%$ tumor remnants have been reported to have favorable prognosis in a neoadjuvant chemotherapy setting (12). Breast cancer guidelines also describe the therapeutic effects of neoadjuvant chemotherapy (13), and histological therapeutic effects are significantly related to prognosis. However, there are many different criteria, and methodological problems still remain (14-16). In cervical cancer, the residual tumor diameter $(<2 \mathrm{~cm})$ has been shown to be a significant prognostic factor for LR cervical cancer post-RT (6). In addition, Ota et al. have reported that the size of the persistent tumor at hysterectomy is important but the prognostic difference is not statistically significant (8). However, these two studies did not mention histological response. Many viable cancer cells would still
Table IV. Site of recurrence.

\begin{tabular}{lccc}
\hline & Locoregional & Locoregional+distant & Distant \\
\hline $\begin{array}{l}\text { No. } \\
\text { Time to recurrence } \\
\text { (months) }\end{array}$ & $2,4,5,6,28$ & 1 & 4 \\
\hline
\end{tabular}

remain in larger residual or recurrent tumors, which would be equivalent to Ef 1 in our study. Histological response shows significance for OS and DFS and seems to be important for prediction of prognosis.

Five patients with enlarged pelvic lymph nodes underwent removal of the lymph nodes, revealing positive nodes. Although positive node was not an independent prognostic factor in our study population, 4 of these patients died of the disease. Previous studies have demonstrated poor prognosis in patients with positive lymph nodes in residual disease post-RT (6-8), suggesting pelvic lymphadenectomy is not always necessary and rarely results in improved survival.

The significance of this study was that we analyzed patients who underwent hysterectomy for LR cervical cancer post-RT at a single institution. However, one of the limitations of this study was its retrospective nature, with a relatively small sample and selection bias. While we made all attempts to acquire complete and accurate data, recall bias and difficulty in data abstraction can affect retrospective chart reviews.

\section{Conclusion}

Adjuvant hysterectomy could be a treatment of choice for LR cervical cancer post-RT. Histological response of cervical cancer is a good indicator that could help select patients who are most likely to benefit from adjuvant hysterectomy.

\section{Conflicts of Interest}

The Authors declare that there are no conflicts of interest regarding the publication of this paper.

\section{Authors' Contributions}

The work presented here was carried out in collaboration among all authors. TN and YA designed the methods, analyzed the data, interpreted the results, and wrote the manuscript. All Authors have read the manuscript, and approved this submission.

\section{Acknowledgements}

The Authors would like to thank Enago (http://www.enago.jp/) for the English language review of this article. 


\section{References}

1 Rose PG, Ali S, Watkins E, Thigpen JT, Deppe G, ClarkePearson DL and Insalaco S; Gynecologic Oncology Group: Long-term follow-up of a randomized trial comparing concurrent single agent cisplatin, cisplatin-based combination chemotherapy, or hydroxyurea during pelvic irradiation for locally advanced cervical cancer: a Gynecologic Oncology Group Study. J Clin Oncol 25(19): 2804-2810, 2007. PMID: 17502627. DOI: $10.1200 /$ JCO.2006.09.4532

2 Stehman FB, Ali S, Keys HM, Muderspach LI, Chafe WE, Gallup DG, Walker JL and Gersell D: Radiation therapy with or without weekly cisplatin for bulky stage $1 \mathrm{~B}$ cervical carcinoma: follow-up of a Gynecologic Oncology Group trial. Am J Obstet Gynecol 197(5): 503.e1-6, 2007. PMID: 17980189. DOI: 10.1016/j.ajog.2007.08.003

3 Toita T, Kato S, Ishikura S, Tsujino K, Kodaira T, Uno T, Hatano K, Sakurai H,Niibe Y, Kazumoto T, Nishimura T, Kitagawa R, Fukutani M, Oguchi M, Umayahara K, Hirashima Y, Aoki Y and Takizawa K; Disease Committee of Radiation Oncology, Japanese Gynecologic Oncology Group: Radiotherapy quality assurance of the Japanese Gynecologic Oncology Group study (JGOG1066): a cooperative phase II study of concurrent chemoradiotherapy for uterine cervical cancer. Int J Clin Oncol 16(4): 379-386, 2011. PMID: 21331768. DOI: 10.1007/s10147-011-0196-4

4 Umayahara K, Takekuma M, Hirashima Y, Noda SE, Ohno T, Miyagi E, Hirahara F, Hirata E, Kondo E, Tabata T, Nagai Y, Aoki Y, Wakatsuki M, Takeuchi M, Toita T, Takeshima N and Takizawa K: Phase II study of concurrent chemoradiotherapy with weekly cisplatin and paclitaxel in patients with locally advanced uterine cervical cancer: The JACCRO GY-01 trial. Gynecol Oncol 140(2): 253-258, 2016. PMID: 26701414. DOI: 10.1016/j.ygyno.2015.12.008

5 Ebina Y, Mikami M, Nagase S, Tabata T, Kaneuchi M, Tashiro H, Mandai M,Enomoto T, Kobayashi Y, Katabuchi H, Yaegashi $\mathrm{N}$, Udagawa Y and Aoki D: Japan Society of Gynecologic Oncology guidelines 2017 for the treatment of uterine cervical cancer. Int J Clin Oncol 24(1): 1-19, 2019. PMID: 30291468. DOI: $10.1007 / \mathrm{s} 10147-018-1351-y$

6 Coleman RL, Keeney ED, Freedman RS, Burke TW, Eifel PJ and Rutledge FN: Radical hysterectomy for recurrent carcinoma of the uterine cervix after radiotherapy. Gynecol Oncol 55(1): 29-35, 1994. PMID: 7959262. DOI: 10.1006/gyno.1994.1242

7 Maneo A, Landoni F, Cormio G, Colombo A and Mangioni C: Radical hysterectomy for recurrent or persistent cervical cancer following radiation therapy. Int J Gynecol Cancer 9(4): 295-301, 1999. PMID: 11240782 . DOI: 10.1046/j.1525-1438.1999.99037.x

8 Ota T, Takeshima N, Tabata T, Hasumi K and Takizawa K: Adjuvant hysterectomy for treatment of residual disease in patients with cervical cancer treated with radiation therapy. Br J Cancer 99(8): 1216-1220, 2008. PMID: 18854823. DOI: 10.1038/sj.bjc.6604619
9 Pervin S, Ruma FI, Rahman K, Ferdous J, Ara R, Abu Syed MM and Goodman A: Adjuvant hysterectomy in patients with residual disease after radiation for locally advanced cervical cancer: a prospective longitudinal study. J Glob Oncol 5: 1-7, 2019. PMID: 30707665. DOI: 10.1200/JGO.18.00157

10 Toita T, Kitagawa R, Hamano T, Umayahara K, Hirashima Y, Aoki Y, Oguchi M, Mikami M and Takizawa K; Cervical Cancer (Vulva Cancer) Committee of Japanese Gynecologic Oncology Group (JGOG): Phase II study of concurrent chemoradiotherapy with high-dose-rate intracavitary brachytherapy in patients with locally advanced uterine cervical cancer: efficacy and toxicity of a low cumulative radiation dose schedule. Gynecol Oncol 126(2): 211-216, 2012. PMID: 22555110. DOI: 10.1016/j.ygyno. 2012.04.036

11 Nagai Y, Toita T, Wakayama A, Nakamoto T, Ooyama T, Tokura A, Inamine M, Kudaka W, Murayama S and Aoki Y: Concurrent chemoradiotherapy with paclitaxel and cisplatin for adenocarcinoma of the cervix. Anticancer Res 32(4): 1475-1479, 2012. PMID: 22493388.

12 Uchida K and Masugi Y: A new grading system of histological response to neoadjuvant therapy for pancreatic cancer. Suizo 31: 812-817, 2016.

13 Subcommittee on criteria for histological treatment effects. Criteria for histological treatment effect. Clinical and Pathological, Breast Cancer Handling Rules. Edited by the Japan Breast Cancer Society. 18 $8^{\text {th }}$ Ed. Tokyo, Kanehara Publishing, 2018, pp. 94-100.

14 Horii R and Akiyama F: Histological assessment of therapeutic response in breast cancer. Breast Cancer 23: 540-545, 2016. PMID: 24173652. DOI: 10.1007/s12282-013-0499-6

15 Rastogi P, Anderson SJ, Bear HD, Geyer CE, Kahlenberg MS, Robidoux A, Margolese RG, Hoehn JL, Vogel VG, Dakhil SR, Tamkus D, King KM, Pajon ER, Wright MJ, Robert J, Paik S, Mamounas EP and Wolmark N: Preoperative chemotherapy: updates of National Surgical Adjuvant Breast and Bowel Project Protocols B-18 and B-27. J Clin Oncol 26: 778-785, 2008. PMID: 18258986. DOI: 10.1200/JCO.2007.15.0235

16 Sahoo S and Lester SC: Pathology of breast carcinomas after neoadjuvant chemotherapy: an overview with recommendations on specimen processing and reporting. Arch Pathol Lab Med 133: 633-642, 2009. PMID: 19391665. DOI: 10.1043/15432165-133.4.633
Received March 29, 2020

Revised April 9, 2020

Accepted April 10, 2020 